Endoscopists are not generally familiar with diseases of the oral cavity. We present here a unique image of the oral cavity in an 83-year-old man who presented with dysarthria. He had been well all his life and never been under the care of a doctor. Computed tomography (CT) of the head revealed a hemorrhage in the left temporal lobe, but this did not enlarge following admission, and the clinical course was uneventful. Fourteen days later, an upper gastrointestinal endoscopy examination was carried out, as anemia was also noted on admission. A large lumen in the oral cavity was observed (Figure 1a). Careful observation identified turbinates at the back of the lumen (Figure $\mathbf{1 b}$ ). It was therefore considered that the lumen was a large fistula into the nasal cavity. The patient had no history of trauma or infection associated with otorhinolaryngological diseases. The head CT on admission did not suggest any tumor invasion or chronic inflammation in the nasal cavity (Figure 2 ). The disease was thought possibly to be an anomaly. Ultimately, the condition was diagnosed as a congenital defect of the hard palate. As the patient had been well and lived a normal life, nobody had noticed the congenital defect before. After rehabilitation, the patient was able to eat and drink again, and moved to another hospital.

Cleft palate is a rare anomaly diagnosed in the field of pediatric surgery [1]. A cleft palate results in abnormal communication between the oral and nasal cavities. Diagnosis at infancy is common [1], but this patient had not been diagnosed and had progressed to adulthood without health problems. It was therefore considered that the original cleft had developed into this large lesion, which was discovered by chance at endoscopy.

\title{
Congenital defect of the hard palate
}
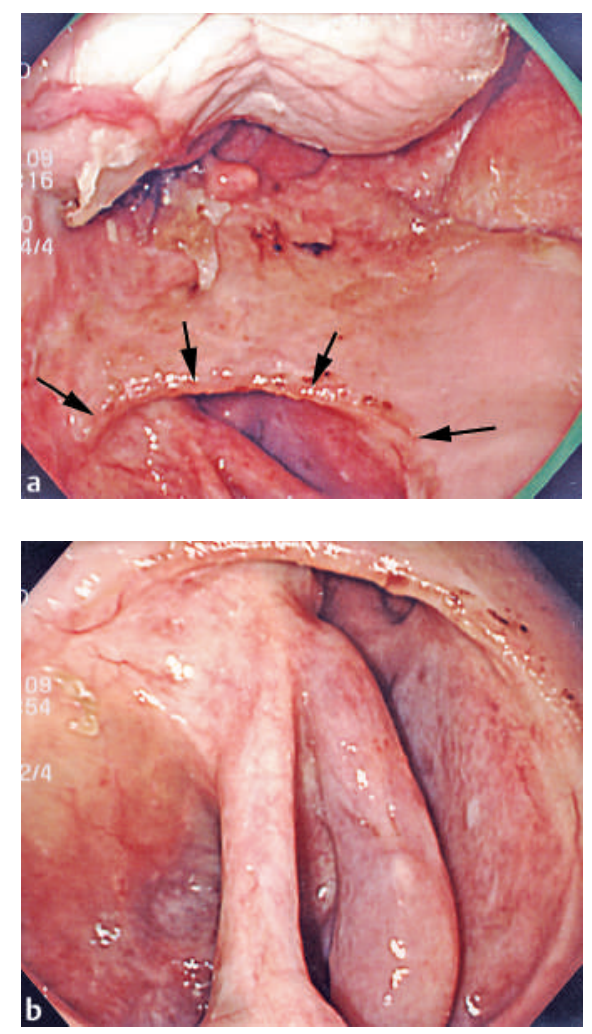

Figure 1 a Endoscopic examination reveals a large lumen in the oral cavity (arrows). b Careful observation identifies turbinates at the back of the lumen.

\section{Endoscopy_UCTN_Code_CCL_1AB_2AB}

\section{S. Tanaka, H. Fujita, M. Iwamuro, J. Kubota, M. Goubaru, T. Ohta}

Dept. of Gastroenterology, National Hospital Organization, Iwakuni Clinical Center, Iwakuni, Japan.

\section{References}

\footnotetext{
${ }^{1}$ Kaufman FL. Managing the cleft lip and palate patient. Pediatr Clin North Am 1991; 38 : $1127-1147$
}

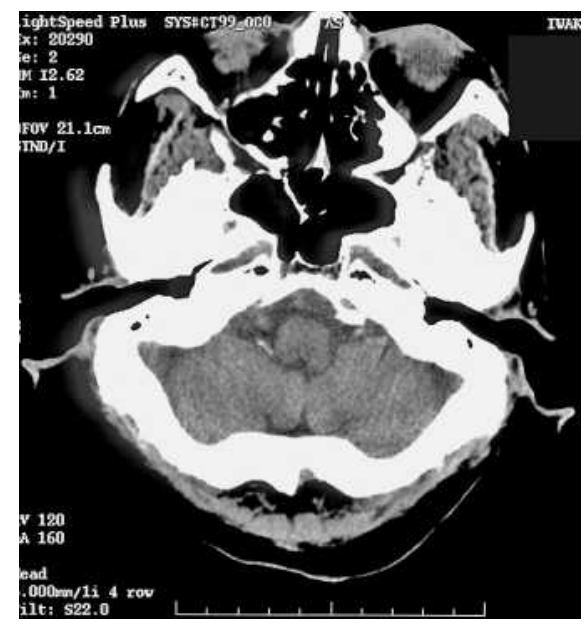

Figure 2 The computed tomogram on admission shows no findings suggesting tumor invasion or chronic inflammation in the nasal cavity.

\section{Corresponding author}

\section{S. Tanaka, M.D.}

Dept. of Gastroenterology

National Hospital Organization

Iwakuni Clinical Center

2-5-1 Kuroiso-cho

Iwakuni 740-0041

Japan

Fax: $\quad+81-827-31-7059$

E-mail: tanaka@iwakuni-nh.go.jp 